\title{
Roles of non-receptor tyrosine kinases in pathogenesis and treatment of depression
}

\author{
John Q. Wang ${ }^{1,2, *}$, Justin D. Derges ${ }^{2}$, Alaya Bodepudi ${ }^{1}$, Nikhila Pokala ${ }^{1}$, Li-Min Mao ${ }^{1}$ \\ ${ }^{1}$ Department of Biomedical Sciences, School of Medicine, University of Missouri-Kansas City, Kansas, MO 64108, USA \\ ${ }^{2}$ Department of Anesthesiology, School of Medicine, University of Missouri-Kansas City, Kansas, MO 64108, USA \\ *Correspondence: wangjq@umkc.edu (John Q. Wang)
}

\section{DOI:10.31083/j.jin2101025}

This is an open access article under the CC BY 4.0 license (https://creativecommons.org/licenses/by/4.0/).

Submitted: 28 July 2021 Revised: 9 August 2021 Accepted: 13 September 2021 Published: 28 January 2022

Major depressive disorder is a chronic psychiatric disease with a high prevalence. Brain mechanisms for depression at cellular and molecular levels are far from clear. Increasing evidence from clinical and preclinical studies reveals critical roles of the non-receptor tyrosine kinase (nRTK) superfamily in the pathophysiology, symptomatology, and therapy of depression. To date, several nRTK members from three nRTK subfamilies, i.e., the Src family kinase (SFK), the Janus tyrosine kinase (JAK) and the focal adhesion kinase (FAK) subfamilies, may connect to the intracellular, intranuclear, and synaptic signaling network linking chronic stress to depression- and anxiety-like behavior. These SFK/JAK/FAK nRTKs are abundantly expressed in the prefrontal cortex and hippocampus, two core limbic regions implicated in depression, and are enriched at synaptic sites. In various acute or chronic animal models of depression, the nRTKs were significantly altered (up- or downregulated) in their phosphorylation, expression, subcellular/subsynaptic distribution, and/or function. Stress that precipitates depressive behavior also influenced the interaction of nRTKs with other signaling molecules and downstream substrates, including ionotropic and metabotropic glutamate receptors. The commonly-used antidepressants showed the ability to alter nRTK activity. In sum, the limbic SFK/JAK/FAK nRTKs are sensitive to stress and undergo drastic adaptations in response to chronic depression. These long-lasting adaptations contribute to the remodeling of signaling network or synaptic plasticity critical for the vulnerability to depression and the therapeutic efficacy of antidepressants.

\section{Keywords}

Tyrosine kinase; Src; Fyn; JAK; FAK; PYK2; Depression; Antidepressant

\section{Introduction}

A tyrosine kinase is a protein enzyme that transfers a phosphoryl group from a nucleoside triphosphate donor, e.g., ATP, to the specific amino acid tyrosine on a protein, i.e., a posttranslational process called phosphorylation. By this liable and reversible process, tyrosine kinases control the expression, subcellular distribution, protein-protein interactions, and function of modified proteins. Tyrosine kinases represent a large family, including receptor tyrosine kinases and non-receptor tyrosine kinases (nRTK). The former are cell surface receptors for growth factors, cytokines, and hormones, which transmit ligand-mediated extracellular signals to the cytoplasm and nucleus. The latter are cytosolic enzymes linking to and thus regulating multiple signal transduction cascades. Most nRTKs are subjected to autophosphorylation or phosphorylation by different nRTKs at tyrosine sites in the activation loop, leading to an increase in enzymatic activity [1]. In the brain, many nRTK members are abundantly expressed in neurons or glial cells. Since some nRTKs are enriched at synaptic sites and are robustly autophosphorylated in response to changing synaptic input, these nRTKs are involved in synaptic plasticity in relation to the strength and efficacy of synaptic transmission or susceptibility of a variety of neurological and neuropsychiatric disorders $[2,3]$.

Major depressive disorder is a neuropsychiatric disorder that negatively affects millions of people. As a mental illness with high prevalence and high rate of treatment-resistance and recurrence, its cause and risk factors have been investigated extensively in preclinical and clinical studies. However, despite all efforts, brain mechanisms underlying depression risk are still obscure. Increasing evidence indicates that chronic exposure to stress seems to be effective in precipitating depression-like behavior in experimental animals. Such long-term incubation of stress is thought to induce neuroadaptations of distinctive signaling pathways in neurons and/or glial cells of brain regions implicated in depression. These adaptive changes then contribute to enduring depressive behavior, although precise molecular mechanisms are far from clear.

nRTKs may connect to the signaling network critical for linking chronic stress to depression-like behavior. It is noted that several subfamilies of nRTKs, including the Src family kinase (SFK), the Janus tyrosine kinase (JAK) and the focal adhesion kinase (FAK), are expressed in the brain at a high level $[4,5]$. Emerging evidence shows that nRTK members from these three families are sensitive to stress that precipitates depression. In fact, in different animal models of depression, acute or chronic stress altered autophosphorylation, expression, subcellular/subsynaptic distribution, and function of these nRTKs in the limbic forebrain regions critical for de- 
Table 1. Changes in nRTK activity in response to depression or antidepressants.

\begin{tabular}{|c|c|c|c|}
\hline nRTK & Changes in phosphorylation or other events & Models of depression or antidepressants & References \\
\hline \multirow[t]{4}{*}{ SFK } & $\begin{array}{l}\text { Increase in Src and Fyn autophosphorylation in the mouse } \\
\text { hippocampus }\end{array}$ & Forced swim & Ohnishi et al. [6] \\
\hline & Decrease in Src phosphorylation in the mouse hippocampus & Postpartum depression & Zhang et al. [8] \\
\hline & Increase in SFK phosphorylation in rat C6 astroglial cells & Antidepressant (amitriptyline) & Abe et al. [7] \\
\hline & $\begin{array}{l}\text { No change in Src/Fyn autophosphorylation, but increase in } \\
\text { Fyn-mGlu5 interactions in the rat striatum }\end{array}$ & Prolonged social isolation & Mao et al. [9] \\
\hline \multirow[t]{4}{*}{ JAK } & $\begin{array}{l}\text { Decrease in JAK2 phosphorylation in the rat orbitofrontal } \\
\text { cortex, which was reversed by ketamine }\end{array}$ & $\begin{array}{l}\text { Chronic intermittent cold and antidepressant (ke- } \\
\text { tamine) }\end{array}$ & Patton et al. [11] \\
\hline & Increase in JAK2 phosphorylation in the rat hippocampus & Antidepressant (venlafaxine) & Saad et al. [10] \\
\hline & $\begin{array}{l}\text { Increase in JAK } 3 \text { phosphorylation in the mouse hippocam- } \\
\text { pus, which was reduced by amitriptyline }\end{array}$ & $\begin{array}{l}\text { High dose of glucocorticosterone and antidepres- } \\
\text { sant (amitriptyline) }\end{array}$ & Gulbins et al. [12] \\
\hline & $\begin{array}{l}\text { Increase in JAK3 phosphorylation in the mouse hippocam- } \\
\text { pus, which was reversed by magnesium isoglycyrrhizinate } \\
\text { and fluoxetine }\end{array}$ & $\begin{array}{l}\text { Lipopolysaccharide and antidepressants (magne- } \\
\text { sium isoglycyrrhizinate and fluoxetine) }\end{array}$ & Jiang et al. [13] \\
\hline \multirow[t]{4}{*}{ FAK } & Increase in FAK mRNA expression in the striatum & Patients with depression & Gao et al. [14] \\
\hline & $\begin{array}{l}\text { Decrease in FAK Y397 phosphorylation and increase in } \\
\text { Pyk2 Y } 402 \text { phosphorylation in the rat PFC }\end{array}$ & Antidepressant (imipramine) & Zalewska et al. [15] \\
\hline & $\begin{array}{l}\text { Decrease in FAK Y397 phosphorylation and increase in } \\
\text { Pyk2 Y } 402 \text { phosphorylation in the rat hippocampus }\end{array}$ & Electroconvulsive shock (therapy for depression) & Kang et al. [16] \\
\hline & Decrease in Pyk2 phosphorylation in the rat lateral septum & Acute restraint stress & Sheehan et al. [17] \\
\hline
\end{tabular}

FAK, focal adhesion kinase; JAK, Janus tyrosine kinase; mGlu, metabotropic glutamate; nRTK, non-receptor tyrosine kinase; Pyk2, proline-

rich tyrosine kinase 2; SFK, Src family kinase.

pression, such as the prefrontal cortex (PFC) and hippocampus. These plastic changes were either pathogenic in promoting the vulnerability for depression or compensatory in normalizing behavioral responses to stress, depending on signaling pathways that nRTKs interact with, downstream substrates of nRTKs, brain regions and circuits, models of depression, etc. Standard antidepressants can also affect the nRTK pathway to exert their therapeutic effects. This review discusses the brain nRTK's sensitivity to depression and potential roles of nRTKs in the pathophysiology and symptomatology of depression with a focus on recent progress. In addition, the potential antidepressant properties of nRTKs and associated signaling pathways are analyzed.

\section{SFK family}

Brain SFKs may be sensitive to stress and may undergo long-term adaptive changes during the development of depression. As a core intracellular signaling pathway, SFKs interact with a large number of substrates, including receptor, signaling, structural, and enzymatic proteins. By interacting with different substrates, SFKs either affect the susceptibility to depression or serve as a dynamic element in a molecular mechanism underlying the effect of antidepressants. Among SFK members enriched in the brain (Src, Fyn, Yes, Lyn, and Lck), Src and Fyn are particularly interesting as they are abundant at synaptic sites and have been demonstrated to have a role in depression. One study found that depressive stress altered autophosphorylation of SFKs in mice, which served as an inducible element to reduce the vulnerability to depression [6]. In details, this study showed that forced swim induced an increase in mouse passive behavior (immobility time), indicating a state of despair or depression. Notably, forced swim also induced the autophosphorylation of SFKs at a conserved pan-Y416 site (leading to activation of SFKs) in the hippocampus (Table 1 Ref. [6-17]). Two key SFK members, Src and Fyn, were affected in this event as autophosphorylation was elevated in both Src and Fyn proteins immunoprecipitated from the hippocampus. Active Src/Fyn then tyrosine-phosphorylated an Ig-superfamily and synapse-rich protein, signal regulatory protein $\alpha(\operatorname{SIRP} \alpha$; also known as SHPS-1 and p84), and increased the binding of SIRP $\alpha$ to its downstream protein, tyrosine protein phosphatase Shp2. This Src/Fyn-SIRP $\alpha$-Shp2 pathway seems to be mobilized to reduce depression risk since mutant mice lacking most of the tyrosine-phosphorylated cytoplasmic region of SIRP $\alpha$ or lacking the SIRP $\alpha$ ligand CD47 manifested the increased immobility time relative to wild-type (WT) mice. Moreover, the tricyclic antidepressants imipramine and desipramine induced tyrosine phosphorylation of SIRP $\alpha$ in the hippocampus of WT mice.

$\mathrm{G}_{\alpha s}$-coupled 5-hydroxytryptamine $6\left(5-\mathrm{HT}_{6}\right)$ receptors may be another target of SFKs. An early study showed that Fyn directly bound to the intracellular $\mathrm{C}$ terminus of $5-\mathrm{HT}_{6}$ receptors [18]. This direct protein-protein interaction enabled $5-\mathrm{HT}_{6}$ receptors to enhance autophosphorylation of Fyn, which thereby activated the mitogen-activated protein kinase (MAPK)/extracellular signal-regulated kinase $1 / 2\left(\right.$ ERK1/2). The role of Fyn in linking $5-\mathrm{HT}_{6}$ receptors to ERK1/2 is noteworthy as the ERK signaling was significantly downregulated in the PFC and hippocampus of depressed 
Fig. 1. A schematic diagram illustrating roles of SFKs in interacting with ionotropic glutamate receptors in relation to depression and antidepressant action. Chronic stress that causes depression-like behavior could enhance autophosphorylation (activation) of synapse-enriched SFKs (mainly Src and Fyn) in the brain regions implicated in the pathogenesis of depression, including the prefrontal cortex and hippocampus. Active Src/Fyn then phosphorylate NMDA receptor GluN2A/2B subunits at a specific set of tyrosine residues, which enhances NMDA receptor activity and promotes the vulnerability to depression. On the other hand, AMPA receptors upon activation could activate a neuron-enriched SFK member Lyn. Active Lyn in turn causes translocation of cytosolic MAPKs to the nucleus to upregulate BDNF gene expression. Enhanced BDNF-TrkB signaling could then participate in molecular events critical for alleviating depression-like behavior. As such, NMDA receptor antagonists and AMPA receptor potentiators are generally considered to possess the antidepressant value and may produce their therapeutic effects partially via a signaling mechanism involving SFKs.

humans and animals and various antidepressants acted in part through normalizing the downregulated ERK activity [19]. Reciprocally, the Fyn binding increased 5- $\mathrm{HT}_{6}$ receptor activity. Given that $5-\mathrm{HT}_{6}$ agonists exhibit antidepressant properties [20] and 5- $\mathrm{HT}_{6}$ receptors have a high affinity for a set of antidepressants [21], Fyn by interacting with 5$\mathrm{HT}_{6}$ receptors could serve as a signaling mechanism for antidepressant effects.

Fyn may interact with neurotrophic factors for the etiology and treatment of depression. Brain-derived neurotrophic factor (BDNF) and its receptor, i.e., the tropomyosin receptor kinase $\mathrm{B}(\mathrm{TrkB})$ receptor, were lowered in their expression or phosphorylation in the PFC and/or hippocampus of depressed humans and animals, and BDNF has been generally shown to have antidepressant properties [19, 22, 23]. A recent study provided evidence supporting that BDNF exerts its antidepressant effects partially via a signaling mechanism involving Fyn. According to Diniz et al. [24], blockade of the angiotensin II receptor 1 (AGTR1) with losartan shifted angiotensin II to interact with AGTR2 receptors. Activated AGTR2 then recruited Fyn to TrkB receptors, leading to transactivation of TrkB receptors. Through the linkage of AGTR2 to TrkB by Fyn, the AGTR1 blocker reduced the immobility time in the forced swim test and is thus considered to have therapeutic potential as a novel class of antidepressants. In addition to BDNF, glial cell line-derived neurotrophic factor (GDNF) is regulated by an SFK-dependent pathway. The antidepressant amitriptyline increased SFK phosphorylation in rat C6 astroglial cells [7]. Amitriptyline also induced GDNF production, which was blocked by SFK inhibitors. Since astrocytes are implicated in processing the effect of antidepressants by producing neurotrophic/growth factors [7, 22], SFKs could contribute to the antidepressant efficacy by mediating the neurotrophic factor production in astrocytes. 
Postpartum depression affects approximately $15 \%$ of mothers after childbirth [25]. In ovariectomized female rats that were treated with pregnancy-associated hormones (estrogen and progesterone) to create a hormone-stimulated pregnancy, abrupt withdrawal of hormone treatment precipitated depression-like behavior, mimicking the postpartum depression in humans [26]. In a similar model of postpartum depression in mice, Src phosphorylation was reduced in the hippocampus [8]. The reduced Src activity, as an initial step, led to a series of subsequent events, i.e., decreased tyrosine phosphorylation of $N$-methyl- ${ }_{\mathrm{D}}$-aspartate (NMDA) receptors (see below), impaired neurogenesis in the hippocampus, and finally depression-like behavior. Consistent with this scenario, systemic administration of the Src inhibitor dasatinib caused depression- and anxiety-like behaviors in hormone-stimulated pregnancy rats [8].

Of note, at present, there remain significant limitations in our ability to investigate molecular mechanisms underlying pathophysiology of human mental illnesses due to the difficulties of convincingly modeling human brain disorders in animals. Major depression is among disorders whose clinical features in humans are difficult to model in animals [27]. Depression includes various sets of symptoms, and a major subset of symptoms that can be measured objectively in rodents include anhedonia, psychomotor behavior, and homeostatic symptoms. Acute stress paradigms such as the forced swim test involve short-term stress applied to normal rodents, which is different from the chronically developed human depression pathology. Moreover, the forced swim test measures the altered behavioral responses to stress, which include but are not limited to depression. Thus, this test may not provide definitive evidence for a depression phenotype [27-29]. Nevertheless, despite the limitations, assays based on acute stress exposure might be useful in initial screens [27]. Future studies will aim to (1) generate useful rodent and invertebrate models with the strong construct (or etiologic), face, and predictive (or pharmacological) validity, and (2) utilize chronic stress models more frequently and broaden the scope of behavioral assays. These endeavors are deemed helpful for promoting analysis of roles of nRTKs in pathogenesis of depression and antidepressant action.

\section{SFK-glutamate receptor interactions in depression}

Glutamatergic transmission is a non-monoamine-based system implicated in depression and antidepressant action [30]. Since SFKs vigorously phosphorylate NMDA receptors in response to changing synaptic input [31, 32], SFKs could modulate NMDA receptors to influence the state of depression and the effect of antidepressants. Functional NMDA receptors are assembled by obligatory GluN1 (formerly known as NR1) and modulatory GluN2 subunits, mainly GluN2A (NR2A) and GluN2B (NR2B) [33]. The GluN2A C-terminal region harbors multiple tyrosine sites phosphorylated by Src and/or Fyn, including Y842, Y1292, Y1325, and Y1387 [34-
36]. Among these sites, Y1325 seems to be particularly significant. Phosphorylation of Y1325 by Src potentiated NMDA receptor channels in medium spiny neurons of the mouse striatum [36]. Forced swim activated SFKs and phosphorylated GluN2A at Y1325 in striatal neurons (Fig. 1). Mutation of Y1325 to phenylalanine (Y1325F) prevented the phosphorylation at this site in transgenic mice in vivo and induced antidepressant-like behavior (less immobile). Thus, the SFKGluN2A coupling links swim stress to behavioral immobility.

In addition to GluN2A, GluN2B is a substrate of SFKs and is implicated in depression (Fig. 1). In mice, forced swim induced parallel increases in phosphorylation of SFKs and GluN2B Y1472 in the mouse hippocampus [6]. This increase in GluN2B Y1472 phosphorylation was absent in Fyn-deficient mice. Thus, Fyn mediated the upregulation of GluN2B Y1472 phosphorylation and this Fyn-GluN2B pathway is considered to be a metabasis processing NMDA receptor plasticity and behavioral immobility in response to forced swim. These findings are consistent with a general view that NMDA receptor antagonism is of an antidepressant value [37, 38]. However, in a different model of depression, the SFK-GluN2B pathway seems to play a different role. In ovariectomized mice, estrogen hormone treatment increased Src and GluN2B phosphorylation in the hippocampus, while withdrawal of hormone treatment induced depression- and anxiety-like behavior and reduced hippocampal Src and GluN2B phosphorylation [8]. Repeated systemic administration of NMDA prevented depression-like behavior, while the GluN2B inhibitor Ro25-6981 or Src inhibitor dasatinib precipitated depression behavior in ovariectomized mice treated with hormones.

The $\alpha$-amino-3-hydroxy-5-methylisoxazole-4-propionic acid (AMPA) receptor was found to signal through SFKs. The SFK member Lyn was physically associated with AMPA receptors in the cerebellum, a brain area implicated in depression [39], and was rapidly activated after stimulation of the receptor [40] (Fig. 1). Active Lyn then recruited MAPKs to increase BDNF expression. This Lyn-MAPK-BDNF pathway is intriguing as it may contribute to the antidepressant efficacy of AMPA receptor potentiators in various animal models of depression [37, 38, 41].

Other glutamate receptors which SFKs interact with are metabotropic glutamate (mGlu) receptors. Among three functional groups of $\mathrm{mGlu}$ receptors, group I mGlu receptors (mGlu1/5) have been most extensively studied for their linkage to depression and the antidepressant property [2]. Group I mGlu receptors are coupled to $\mathrm{G} \alpha \mathrm{q}$ proteins and activation of them activates phospholipase $\mathrm{C} \beta 1$, which in turn hydrolyzes phosphoinositide into inositol-1,4,5-triphosphate $\left(\mathrm{IP}_{3}\right)$ and diacylglycerol (DAG). Through $\mathrm{IP}_{3}$ and $\mathrm{DAG}$, mGlu1/5 receptors actively modulate various cellular and synaptic events [42]. It has been found that mGlu5 receptors in the rat brain were tyrosine-phosphorylated [43]. Recently, mGlu5 receptors were found to be sensitive to depression as this receptor underwent adaptive upregulation in its 
expression in the adult rat striatum following prolonged social isolation [44]. This upregulation appears to be partially mediated by a signaling mechanism involving SFKs based on the findings that (1) the SFK member Fyn interacted with striatal mGlu5 receptors, (2) this Fyn-mGlu5 interaction was increased following social isolation, and (3) the SFK inhibitor PP2 reduced the upregulation of mGlu5 expression in the striatum of socially isolated rats [9]. Of note, antidepressant activity has been seen following the use of antagonists and negative allosteric modulators selective for $\mathrm{mGlu} 5$ receptors $[45,46]$.

In summary, SFKs exhibit the ability to interact with ionotropic glutamate receptors. By influencing NMDA and AMPA receptors, SFKs play a role in the antidepressant property of NMDA receptor antagonists and AMPA receptor potentiators. In addition, SFKs were recently found to interact with mGlu receptors. By interacting with mGlu5 receptors, Fyn facilitates the upregulation of striatal mGlu5 expression in socially isolated rats showing depression-like behavior. It is possible that the Fyn-mGlu5 interaction serves as an essential element in a molecular mechanism linking social isolation to depressive behavior.

\section{JAK family}

The JAK family of nRTKs contains four members: JAK1, JAK2, JAK3, and tyrosine kinase 2 (TYK2). The canonical JAK signaling cascade is initiated by ligand-mediated activation of a gp130 receptor subunit, which promotes autophosphorylation of JAKs. Active JAKs induce phosphorylation of transcription factors known as signal transducers and activators of transcription (STAT). As a key pleiotropic intracellular cascade expressed in the mammalian brain, JAKs and STATs link various signals from cytokines and growth factors to nuclear gene expression and to multiple types of synaptic plasticity. The JAK/STAT pathway is known to play significant roles in immune and inflammatory responses. Increasing evidence shows that neuroinflammation is involved in the pathogenesis of depression, and the JAK/STAT pathway may be related to the efficacy of antidepressants [47]. Indeed, several studies show that the JAK2 signaling is critical for antidepressants. Saad et al. [10] found that the antidepressant venlafaxine after chronic administration markedly decreased immobility time in depressed female rats following ovariectomy. In parallel, venlafaxine enhanced phosphorylation of JAK2, STAT5, and ERK1/2 and expression of BDNF in the hippocampus. Thus, venlafaxine may exert its antidepressant effect partially though activating the JAK2/STAT5 pathway. The JAK2 signaling may also contribute to the efficacy of ketamine, a rapid-acting antidepressant. Patton et al. [11] reported that a single injection of ketamine rescued cognitive impairments in depression induced by chronic intermittent cold (CIC) in rats. CIC stress reduced JAK2 phosphorylation in the orbitofrontal cortex, which was reversed by ketamine. The JAK2 inhibitor AG490 prevented the ketamine-induced beneficial behavioral effect and reduc- tion of local field potentials in the orbitofrontal cortex evoked by stimulation of excitatory thalamic afferents. In cortical neuronal cultures, JAK2 inhibition prevented the ketaminestimulated expression of the neural plasticity-related protein Arc. In addition, inhibiting interleukin-6 or its downstream JAK/STAT pathway in the orbitofrontal cortex impaired rat cognitive flexibility [48]. These results support JAK2 as a player in the signaling pathway processing the therapeutic effect of ketamine. Consistent with this, erythropoietin, a hematopoietic growth factor, seems to produce the antidepressant-like effect. The brain is among the organs where significant erythropoietin production and secretion occur. The erythropoietin receptor is expressed in multiple regions of the central nervous system, including the cortex, hypothalamus, and hippocampus [49]. The nonhematopoietic effect of erythropoietin on depression may be attributed to its binding to the erythropoietin receptor, which results in activation of the principal downstream JAK2 signaling cascade [49]. However, in another study, forced swim increased STAT3 mRNA expression and protein phosphorylation in the rat PFC and hippocampus, which was reversed by antidepressants $N$-acetylcysteine and fluoxetine [50]. Thus, normalizing upregulated STAT3 responses to stress may be involved in mediating the effect of these antidepressants.

JAK3 is also connected to the signaling network related to depression. Stress induced by high doses of glucocorticosterone triggered phosphorylation (activation) of JAK3 in the hippocampus and caused depression-like behavior in mice [12]. The JAK3 inhibitor IV after repeated systemic administration improved behavior of depressed mice, and the antidepressant amitriptyline reduced the stress-induced phosphorylation of JAK3. Similarly, lipopolysaccharide (LPS), the main component of outer membrane of gram-negative bacteria which knowingly triggers the inflammatory cascade and induces depression in murine models [51], elevated phosphorylation of JAK3 and STAT3 in the mouse hippocampus [13]. Magnesium isoglycyrrhizinate and fluoxetine with the antidepressant value reversed the responses of JAK3 and STAT3 to LPS. These data suggest that the JAK3/STAT3 pathway in the hippocampus is upregulated in response to stress. Antidepressants may produce their effects by limiting the JAK3/STAT3 signaling.

In summary, evidence is available to support the role of the JAK2 signaling in processing effects of antidepressants. Inhibition of JAK2 prevents the antidepressant action of ketamine. In addition, antidepressants are able to reverse an increase in phosphorylation of hippocampal JAK3 in response to stress.

\section{FAK familly}

The FAK family comprises two homologous members, FAK (also known as protein tyrosine kinase 2, PTK2) and proline-rich tyrosine kinase 2 (Pyk2) also named PTK2B or FAK2. FAK is activated by integrin engagement or other cell surface receptors. Active FAK serves as a signal- 
ing/scaffolding integrator for forming multimolecular complexes controlling migration, synapse formation, and neurite growth throughout brain development $[52,53]$. As one of the nRTKs present in the postsynaptic density (PSD) microdomain [5], FAK participates in the formation of synaptic plasticity in response to changing synaptic input in adulthood brains $[52,54]$. As such, FAK is likely to orchestrate structural and functional events at synaptic sites in response to depression. Indeed, one study found that FAK mRNA expression as detected by microarray analysis was enhanced in the striatum of patients with depression, and FAK gene thus might be among novel biomarkers for the depression diagnosis [14]. In a study with a commonly-used antidepressant, chronic imipramine administration reduced FAK Y397 autophosphorylation in the rat PFC [15]. Electroconvulsive shock, an effective therapy for depression, also induced a rapid and transient dephosphorylation of FAK at Y397 in the rat hippocampus [16]. These results suggest that FAK is significant in the pathogenesis of depression and antidepressant action.

Like FAK, Pyk2 is expressed in neurons. In fact, its expression in the brain is most prominent [55]. Pyk2 noticeably clusters to the PSD $[56,57]$ and thus can vigorously regulate synaptic plasticity [56-60]. As a $\mathrm{Ca}^{2+}$-sensitive kinase, Pyk 2 is activated by intracellular $\mathrm{Ca}^{2+}$ signals and achieves its full activation through phosphorylation by Src/Fyn [61]. Sheehan et al. [17] found that Pyk2 is highly expressed in the rat lateral septum, an area critical for depression and antidepressant drugs [62]. Acute restraint stress for one hour decreased Pyk2 Y402 and ERK phosphorylation in the rat lateral septum, where enhancing Pyk2 expression via a local virusmediated gene transfer produced the antidepressant effect [17]. Enhanced Pyk2 Y402 phosphorylation was also seen in the rat PFC and/or hippocampus following imipramine treatment [15] or electroconvulsive shock [16]. Thus, Pyk2 seems to undergo downregulation in its activity in response to stress [63]. Antidepressant therapy is able to increase Pyk2 activity to alleviate depression-like behavior.

In different models of depression, Pyk2 activity seems to be a catalyst for depression. Knockout of Pyk2 in mice reduced depression risk in a chronic unpredictable mild stress model of depression [64]. In Pyk2 knockout mice, stress-induced changes in dendritic spine morphology and NMDA receptor phosphorylation and expression were prevented in the amygdala. In another study, chronic restraint stress ( $6 \mathrm{~h}$ a day for 21 days) that precipitated depression induced subcellular redistribution of the active form of Pyk2 (Y402-phosphorylated) in mouse hippocampal pyramidal neurons, while total Y402-phospho-Pyk2 protein levels remained unchanged [65]. This redistribution enabled Pyk2 to phosphorylate a nuclear pore complex protein, nucleoporin p62 (NUP62), at a Pyk2-dependent site (Y422) and thereby promoted ubiquitination and proteasomal degradation of NUP62. The loss of NUP62 in this way may be pathogenic in deteriorating dendritic architecture associated with depression and cognitive impairments.

Altogether, both FAK and Pyk2 are present at synaptic sites and actively regulate synaptic transmission and plasticity. Depending on the animal model of depression, phosphorylation and activity of FAK and Pyk2 in depression-related brain regions are either up- or downregulated by stress. Altered FAK and Pyk2 activity is critical for the development of depression. Antidepressants could achieve their effects partially by normalizing FAK and Pyk2 signaling pathways.

\section{Conclusions}

In this review, we summarized roles of nRTKs in depression and antidepressant action. Available data from clinical and mainly preclinical studies show that nRTKs are linked to the signaling network relevant to depression. Several nRTK members from SFK, JAK, and FAK families were altered in their expression, subcellular distribution, and function in the limbic brain regions (PFC and hippocampus) implicated in depression by acute and especially chronic stress exposure. The SFK interaction with glutamate receptors was also sensitive to stress which promoted depression risk. These plastic changes in the nRTK pathway and associated signaling molecules constitute a biochemical basis for the remodeling of synaptic transmission and plasticity critical for either the pathophysiology of major depressive disorder or a recovery process from depression. In addition, the nRTK pathway is active in processing the efficacy of various antidepressants. Through a signaling mechanism involving nRTKs, antidepressants exert their action in improving depression-like behavior.

Evidently, it is still at an infant stage for the investigation of linkage between nRTKs and depression. Future studies will aim to explore and characterize systematic responses of $\mathrm{nRTKs}$ to stress in broad brain regions and circuits. In these studies, new nRTK members and new substrates of nRTKs may be identified as essential elements or biochemical markers in the signaling network critical for determining the vulnerability of depression. These new elements together with nRTK members that have been demonstrated to be sensitive to depressive stress may also be the targets of antidepressants and act to mediate the effect of antidepressant drugs. Of note, given a large number of nRTKs and their interacting signaling molecules, dynamic crosstalk is expected among these signaling regulators at different levels. It is thus intriguing to explore whether and how the crosstalk occurs and operates coherently to control cellular, synaptic, circuit, and behavioral responses to stress or antidepressants. Finally, at present, clinical studies and trials that target nRTKs in terms of molecular mechanisms and treatment of depression are limited. More clinical studies are expected to be carried out following the elucidation of nRTK-dependent mechanisms underlying the etiology of depression and the efficacy of antidepressants in preclinical experiments. 


\section{Abbreviations}

AGTR, angiotensin II receptor; AMPA, $\alpha$-amino-3hydroxy-5-methylisoxazole-4-propionic acid; BDNF, Brainderived neurotrophic factor; CIC, chronic intermittent cold; DAG, diacylglycerol; ERK, extracellular signal-regulated kinase; FAK, focal adhesion kinase; GDNF, glial cell linederived neurotrophic factor; $5-\mathrm{HT}_{6}, 5$-hydroxytryptamine 6; $\mathrm{IP}_{3}$, inositol-1,4,5-triphosphate; JAK, Janus tyrosine kinase; LPS, lipopolysaccharide; MAPK, mitogen-activated protein kinase; mGlu, metabotropic glutamate; NMDA, $N$-methyl$D$-aspartate; nRTK, non-receptor tyrosine kinase; NUP62, nucleoporin p62; PFC, prefrontal cortex; PSD, postsynaptic density; PTK2, protein tyrosine kinase 2; Pyk2, proline-rich tyrosine kinase 2; SFK, Src family kinase; $\operatorname{SIRP} \alpha$, signal regulatory protein $\alpha$; STAT, signal transducers and activators of transcription; TrkB, tropomyosin receptor kinase B; TYK2, tyrosine kinase 2; WT, wild-type.

\section{Author contributions}

JQW and LMM initiated the idea for the article. JQW, JDD, AB, NP, and LMM conducted the literature search and drafted and critically revised the work.

\section{Ethics approval and consent to participate Not applicable.}

\section{Acknowledgement}

The authors want to thank the NIH for the research grant that promotes the production of this and other publications.

\section{Funding}

The work by the authors discussed in this article was supported by an NIH grant (R01 MH061469).

\section{Conflict of interest}

The authors declare no conflict of interest.

\section{References}

[1] Reinecke J, Caplan S. Endocytosis and the Src family of nonreceptor tyrosine kinases. Biomolecular Concepts. 2014; 5: 143155.

[2] Mao L, Wang JQ. Tyrosine phosphorylation of glutamate receptors by non-receptor tyrosine kinases: roles in depression-like behavior. Neurotransmitter. 2016; 3: e1118.

[3] Mao L, Geosling R, Penman B, Wang JQ. Local substrates of nonreceptor tyrosine kinases at synaptic sites in neurons. Sheng $\mathrm{Li}$ Xue Bao. 2017; 69: 657-665. (In Chinese)

[4] Kalia LV, Gingrich JR, Salter MW. Src in synaptic transmission and plasticity. Oncogene. 2004; 23: 8007-8016.

[5] Bongiorno-Borbone L, Kadaré G, Benfenati F, Girault J. FAK and PYK2 interact with SAP90/PSD-95-Associated Protein-3. Biochemical and Biophysical Research Communications. 2005; 337 : 641-646.

[6] Ohnishi H, Murata T, Kusakari S, Hayashi Y, Takao K, Maruyama $\mathrm{T}$, et al. Stress-evoked tyrosine phosphorylation of signal regulatory protein $\alpha$ regulates behavioral immobility in the forced swim test. Journal of Neuroscience. 2010; 30: 10472-10483.

[7] Abe H, Kajitani N, Okada-Tsuchioka M, Omori W, Yatsumoto M, Takebayashi M. Antidepressant amitriptyline-induced matric metalloproteinase- 9 activation is mediated by Src family tyrosine kinase, which leads to glial cell line-derived neurotrophic factor mRNA expression in rat astroglial cells. Neuropsychopharmacology Reports. 2019; 39: 156-163.

[8] Zhang Z, Hong J, Zhang S, Zhang T, Sha S, Yang R, et al. Postpartum estrogen withdrawal impairs hippocampal neurogenesis and causes depression- and anxiety-like behaviors in mice. Psychoneuroendocrinology. 2016; 66: 138-149.

[9] Mao L, Wang JQ. Linkage of Non-receptor Tyrosine Kinase Fyn to mGlu5 Receptors in Striatal Neurons in a Depression Model. Neuroscience. 2020; 433: 11-20.

[10] Saad MA, El-Sahar AE, Sayed RH, Elbaz EM, Helmy HS, Senousy MA. Venlafaxine Mitigates Depressive-Like Behavior in Ovariectomized Rats by Activating the EPO/EPOR/JAK2 Signaling Pathway and Increasing the Serum Estradiol Level. Neurotherapeutics. 2019; 16: 404-415.

[11] Patton MS, Lodge DJ, Morilak DA, Girotti M. Ketamine Corrects Stress-Induced Cognitive Dysfunction through JAK2/STAT3 Signaling in the Orbitofrontal Cortex. Neuropsychopharmacology. 2018 ; 42: 1220-1230.

[12] Gulbins A, Grassmé H, Hoehn R, Kohnen M, Edwards MJ, Kornhuber J, et al. Role of Janus-Kinases in Major Depressive Disorder. Neuro-Signals. 2019; 24: 71-80.

[13] Jiang W, Chen Q, Li P, Lu Q, Pei X, Sun Y, et al. Magnesium Isoglycyrrhizinate attenuates lipopolysaccharide-induced depressivelike behavior in mice. Biomedicine \& Pharmacotherapy. 2017; 86: 177-184.

[14] Gao L, Gao Y, Xu E, Xie J. Microarray Analysis of the Major Depressive Disorder mRNA Profile Data. Psychiatry Investigation. 2015; 12: 388-396.

[15] Zalewska T, Bielawski A, Stanaszek L, Wieczerzak K, ZiemkaNałęcz M, Nalepa I. Imipramine administration induces changes in the phosphorylation of FAK and PYK2 and modulates signaling pathways related to their activity. Biochimica Et Biophysica Acta. 2016; 1860: 424-433.

[16] Kang UG, Jun SJ, Yoon SC, Jeon S, Park J, Chung CK, et al. Differential regulation of FAK and PYK2 tyrosine phosphorylation after electroconvulsive shock in the rat brain. Neuroscience Letters. 2004; 363: 134-138.

[17] Sheehan TP, Neve RL, Duman RS, Russell DS. Antidepressant effect of the calcium-activated tyrosine kinase Pyk2 in the lateral septum. Biological Psychiatry. 2003; 54: 540-551.

[18] Yun H, Kim S, Kim H, Kostenis E, Kim JI, Seong JY, et al. The novel cellular mechanism of human 5-HT6 receptor through an interaction with Fyn. Journal of Biological Chemistry. 2007; 282: 5496-5505.

[19] Wang JQ, Mao L. The ERK Pathway: Molecular Mechanisms and Treatment of Depression. Molecular Neurobiology. 2019; 56: 6197-6205.

[20] Wesołowska A. Potential role of the 5-HT6 receptor in depression and anxiety: an overview of preclinical data. Pharmacological Reports. 2011; 62: 564-577.

[21] Roth BL, Craigo SC, Choudhary MS, Uluer A, Monsma FJ, Shen $Y$, et al. Binding of typical and atypical antipsychotic agents to 5-hydroxytryptamine-6 and 5-hydroxytryptamine-7 receptors. Journal of Pharmacology and Experimental Therapeutics. 1994; 268: 1403-1410.

[22] Schmidt HD, Banasr M, Duman RS. Future antidepressant targets: neurotrophic factors and related signaling cascades. Drug Discovery Today: Therapeutic Strategies. 2008; 5: 151-156.

[23] Castrén E. Neurotrophins and psychiatric disorders. Handbook of Experimental Pharmacology. 2014; 220: 461-479.

[24] Diniz CRAF, Casarotto PC, Fred SM, Biojone C, Castrén E, Joca SRL. Antidepressant-like effect of losartan involves TRKB transactivation from angiotensin receptor type 2 (AGTR2) and recruitment of FYN. Neuropharmacology. 2019; 135: 163-171.

[25] Frieder A, Fersh M, Hainline R, Deligiannidis KM. Pharmacotherapy of Postpartum Depression: Current Approaches and Novel Drug Development. CNS Drugs. 2019; 33: 265-282. 
[26] Galea LA, Wide JK, Barr AM. Estradiol alleviates depressive-like symptoms in a novel animal model of post-partum depression. Behavioural Brain Research. 2001; 122: 1-9.

[27] Nestler EJ, Hyman SE. Animal models of neuropsychiatric disorders. Nature Neuroscience. 2010; 13: 1161-1169.

[28] Commons KG, Cholanians AB, Babb JA, Ehlinger DG. The Rodent Forced Swim Test Measures Stress-Coping Strategy, not Depression-like Behavior. ACS Chemical Neuroscience. 2018; 8: 955-960.

[29] Armario A. The forced swim test: Historical, conceptual and methodological considerations and its relationship with individual behavioral traits. Neuroscience \& Biobehavioral Reviews. 2021; 128: 74-86.

[30] Sanacora G, Treccani G, Popoli M. Towards a glutamate hypothesis of depression: an emerging frontier of neuropsychopharmacology for mood disorders. Neuropharmacology. 2012; 62: 63-77.

[31] Groveman BR, Feng S, Fang X, Pflueger M, Lin S, Bienkiewicz EA, et al. The regulation of $N$-methyl- ${ }_{D}$-aspartate receptors by Src kinase. FEBS Journal. 2012; 279: 20-28.

[32] Trepanier CH, Jackson MF, MacDonald JF. Regulation of NMDA receptors by the tyrosine kinase Fyn. FEBS Journal. 2012; 279: 1219.

[33] Traynelis SF, Wollmuth LP, McBain CJ, Menniti FS, Vance KM, Ogden KK, et al. Glutamate receptor ion channels: structure, regulation, and function. Pharmacological Reviews. 2010; 62: 405496.

[34] Lau L, Huganir RL. Differential Tyrosine Phosphorylation of $N$-Methyl- $D$-aspartate Receptor Subunits. Journal of Biological Chemistry. 1995; 270: 20036-20041.

[35] Yang M, Leonard JP. Identification of mouse NMDA receptor subunit NR2a C-terminal tyrosine sites phosphorylated by coexpression with v-Src. Journal of Neurochemistry. 2001; 77: 580588.

[36] Taniquchi S, Nakazawa T, Tanimura A, Kiyama Y, Tezuka T, Watabe AM, et al. Involvement of NMDAR2A tyrosine phosphorylation in depression-related behavior. European Molecular Biology Organization. 2009; 28: 3717-3729.

[37] Aleksandrova LR, Phillips AG, Wang YT. Antidepressant effects of ketamine and the roles of AMPA glutamate receptors and other mechanisms beyond NMDA receptor antagonism. Journal of Psychiatry \& Neuroscience. 2018; 42: 222-229.

[38] Jaso B, Niciu M, Iadarola N, Lally N, Richards E, Park M, et al. Therapeutic Modulation of Glutamate Receptors in Major Depressive Disorder. Current Neuropharmacology. 2016; 15: 57-70.

[39] Depping MS, Schmitgen MM, Kubera KM, Wolf RC. Cerebellar Contributions to Major Depression. Frontiers in Psychiatry. 2020; 9: 634.

[40] Hayashi T, Umemori H, Mishina M, Yamamoto T. The AMPA receptor interacts with and signals through the protein tyrosine kinase Lyn. Nature. 1999; 397: 72-76.

[41] O'Neill MJ, Bleakman D, Zimmerman DM, Nisenbaum ES. AMPA receptor potentiators for the treatment of CNS disorders. Current Drug Targets. CNS and Neurological Disorders. 2004; 3: 181-194.

[42] Niswender CM, Conn PJ. Metabotropic glutamate receptors: physiology, pharmacology, and disease. Annual Review of Pharmacology and Toxicology. 2010; 50: 295-322.

[43] Orlando LR, Dunah AW, Standaert DG, Young AB. Tyrosine phosphorylation of the metabotropic glutamate receptor mGluR5 in striatal neurons. Neuropharmacology. 2003; 43: 161-173.

[44] Mao L, Wang JQ. Alterations in mGlu5 receptor expression and function in the striatum in a rat depression model. Journal of Neurochemistry. 2018; 145: 287-298.

[45] Pałucha-Poniewiera A, Wierońska JM, Brański P, Burnat G, Chruścicka B, Pilc A. Is the mGlu5 receptor a possible target for new antidepressant drugs? Pharmacological Reports. 2014; 65: 1506-1511.

[46] Chaki S, Fukumoto K. MGlu receptors as potential targets for novel antidepressants. Current Opinion in Pharmacology. 2018;
38: 24-30.

[47] Shariq AS, Brietzke E, Rosenblat JD, Pan Z, Rong C, Ragguett R, et al. Therapeutic potential of JAK/STAT pathway modulation in mood disorders. Reviews in the Neurosciences. 2019; 30: 1-7.

[48] Donegan JJ, Girotti M, Weinberg MS, Morilak DA. A novel role for brain interleukin-6: facilitation of cognitive flexibility in rat orbitofrontal cortex. Journal of Neuroscience. 2014; 34: 953-962.

[49] Ma C, Cheng F, Wang X, Zhai C, Yue W, Lian Y, et al. Erythropoietin Pathway: a Potential Target for the Treatment of Depression. International Journal of Molecular Sciences. 2016; 17: 677.

[50] Al-Samhari MM, Al-Rasheed NM, Al-Rejaie S, Al-Rasheed NM, Hasan IH, Mahmoud AM, et al. Possible involvement of the JAK/STAT signaling pathway in N-acetylcysteine-mediated antidepressant-like effects. Experimental Biology and Medicine. 2016; 241: 509-518.

[51] Zhang K, Liu J, You X, Kong P, Song Y, Cao L, et al. P2X7 as a new target for chrysophanol to treat lipopolysaccharide-induced depression in mice. Neuroscience Letters. 2016; 613: 60-65.

[52] Armendáriz BG, Masdeu MDM, Soriano E, Ureña JM, Burgaya F. The diverse roles and multiple forms of focal adhesion kinase in brain. European Journal of Neuroscience. 2015; 40: 3573-3590.

[53] Navarro AI, Rico B. Focal adhesion kinase function in neuronal development. Current Opinion in Neurobiology. 2015; 27: 89-95.

[54] Monje FJ, Kim E, Pollak DD, Cabatic M, Li L, Baston A, et al. Focal adhesion kinase regulates neuronal growth, synaptic plasticity and hippocampus-dependent spatial learning and memory. Neuro-Signals. 2012; 20: 1-14.

[55] Menegon A, Burgaya F, Baudot P, Dunlap DD, Girault JA, Valtorta F. FAK+ and PYK2/CAKbeta, two related tyrosine kinase highly expressed in the central nervous system: similarities and differences in the expression pattern. European Journal of Neuroscience. 1999; 11: 3777-3788.

[56] Bartos JA, Ulrich JD, Li H, Beazely MA, Chen Y, Macdonald JF, et al. Postsynaptic clustering and activation of Pyk2 by PSD-95. Journal of Neuroscience. 2010; 30: 449-463.

[57] Lee S, Salazar SV, Cox TO, Strittmatter SM. Pyk2 signaling through Graf1 and PhoA GTPase is required for amyloid- $\beta$ oligomer-triggered synapse loss. Journal of Neuroscience. 2019; 39: 1910-1929.

[58] Huang Y, Lu W, Ali DW, Pelkey KA, Pitcher GM, Lu YM, et al. CAKbeta/Pyk2 kinase is a signaling link for induction of longterm potentiation in CA1 hippocampus. Neuron. 2001; 29: 485496.

[59] Hsin H, Kim MJ, Wang C, Sheng M. Proline-rich tyrosine kinase 2 regulates hippocampal long-term depression. Journal of Neuroscience. 2010; 30: 11983-11993.

[60] Giralt A, Brito V, Chevy Q, Simonnet C, Otsu Y, Cifuentes-Díaz $\mathrm{C}$, et al. Pyk2 modulates hippocampal excitatory synapses and contributes to cognitive deficits in a Huntington's disease model. Nature Communications. 2018; 8: 15592.

[61] Murphy JM, Jeong K, Lim STS. FAK family kinases in vascular diseases. International Journal of Molecular Sciences. 2020; 21: 3630.

[62] Sheehan TP, Chambers RA, Russell DS. Regulation of affect by the lateral septum: implications for neuropsychiatry. Brain Research. Brain Research Reviews. 2004; 46: 71-117.

[63] Kulikova E, Kulikov A. Striatal-enriched Tyrosine Protein Phosphatase (STEP) in the Mechanisms of Depressive Disorders. Current Protein \& Peptide Science. 2017; 18: 1152-1162.

[64] Montalban E, Al-Massadi O, Sancho-Balsells A, Brito V, de Pins $\mathrm{B}$, Alberch J, et al. Pyk2 in the amygdala modulates chronic stress sequelae via PSD-95-related micro-structural changes. Translational Psychiatry. 2019; 9: 3.

[65] Kinoshita Y, Hunter RG, Gray JD, Mesias R, McEwen BS, Benson DL, et al. Role of NUP62 depletion and PYK2 redistribution in dendritic retraction resulting from chronic stress. Proceedings of the National Academy of Sciences USA. 2014; 111: 16130-16135. 